\title{
B7-H3 inhibits apoptosis of gastric cancer cell by interacting with Fibronectin
}

\author{
Meiyun Sun ${ }^{1,2 \#}$, Jinjing Xie ${ }^{1,2 \#, ~ D o n g z e ~ Z h a n g 2 ~}{ }^{2}$ Chunyang Chen², Simin $\operatorname{Lin}^{1,2}$, Yan Chen ${ }^{2 \bowtie}$, Guangbo \\ Zhang $^{1,2}$ - \\ 1. Medical College of Soochow University, 199 Ren ai Road, Suzhou, Jianosu Province, 215100, China. \\ 2. Jiangsu Institute of Clinical Immunology, The First Affiliated Hospital of Soochow University, 708 Ren min Road, Suzhou, Jiangsu Province, 215100, China. \\ \#These authors contributed equally to this work. \\ $\triangle$ Corresponding author: Yan Chen (E-mail: walves@suda.edu.cn) or Guangbo Zhang (E-mail: zhanggbsuzhou@hotmail.com), 708 Ren min Road, Suzhou, \\ Jiangsu Provine, 215100, China.
}

(c) The author(s). This is an open access article distributed under the terms of the Creative Commons Attribution License (https://creativecommons.org/licenses/by/4.0/). See http://ivyspring.com/terms for full terms and conditions.

Received: 2021.02.09; Accepted: 2021.10.06; Published: 2021.11.08

\begin{abstract}
Anti-apoptosis has been widely accepted as a hallmark of malignancy. B7-H3, a type I transmembrane protein, plays a key role in anti-apoptosis and immune escape, but its regulation during cancer development remains unclear. To investigate how the effect of anti-apoptosis is regulated by $\mathrm{B} 7-\mathrm{H} 3$ in gastric cancer, we stably knocked down B7-H3 gene by shRNA in MGC-803 and MKN-45 cells. The correlation between B7-H3 and Fibronectin (FN) expression were investigated by bioinformatics in public data from TCGA (The Cancer Genome Atlas). Here, we reported that B7-H3 expression is positively correlated with $\mathrm{FN}$ in clinical gastric cancer samples, and B7-H3 promoted adhesion and inhibited apoptosis of gastric cancer cell through an FN-dependent pathway. Mechanistically, B7-H3 interacted with FN and subsequently activated PI3K/AKT signaling pathway, a critical mediator of oncogenic signaling. In addition, exogenous $\mathrm{FN}$ could inhibit the expression of pro-apoptosis-related proteins such as Caspase 3, Caspase 8, Caspase 9, Bax, p53, Apaf-1 and Cleaved PARP, and upregulated the levels of signal molecule p-PI3K, p-AKT and anti-apoptotic proteins $\mathrm{Bcl}-2$ in $\mathrm{B} 7-\mathrm{H} 3$ high group, as compared with those in $\mathrm{B} 7-\mathrm{H} 3$ low group. In conclusion, we here for the first time revealed that $\mathrm{B} 7-\mathrm{H} 3$ inhibits apoptosis of gastric cancer cell through regulation of $\mathrm{FN}$-mediated PI3K/AKT signaling pathways.
\end{abstract}

Key words: Gastric cancer; B7-H3; Fibronectin; adhesion; apoptosis

\section{Introduction}

As one of most common malignant tumors, gastric cancer (GC) is presently mainly treated by surgery, chemotherapy, radiotherapy and immunotherapy, but these treatments still could not significantly improve patient's survival [1], and this also points to the need to explore the biological characteristics of gastric cancer.

B7-H3, which is a type I transmembrane protein of the B7 family, consist of two subtypes 2Ig B7-H3 and 4Ig B7-H3 [2]. B7-H3 was discovered in 2001 by Chapoval et al. [3], and reported as a co-stimulator molecule to enhance cytotoxic T lymphocyte (CTL) activity in vitro and stimulate the growth of $\mathrm{CD}^{+}$and $\mathrm{CD}^{+} \mathrm{T}$ cells[4]. Liu et al. found that B7-H3 inhibits the $\mathrm{T}$ cell response by promoting the secretion of the immunosuppressive cytokine IL-10 [5]. Moreover,
B7-H3 affects T cells and other immune cells such as NK cells [6]. In mouse model of liver cancer and EL-4 lymphoma, tumors expressing B7-H3 plasmid were significantly reduced, partly due to increased NK cell infiltration [7].

In addition, as a tumor-associated antigen, B7-H3 plays a key role in tumor progress. B7-H3 is high-expressed in gastric cancer, colorectal cancer, prostate cancer, kidney cancer, lung cancer, and breast cancer $[4,8,9]$. Previous reports have shown that overexpression of $\mathrm{B} 7-\mathrm{H} 3$ contributes to immune evasion of tumors and promotes metastasis, thereby leading to a poor prognosis [10]. In oral squamous cell carcinoma, overexpressed $\mathrm{B} 7-\mathrm{H} 3$ is positively associated with a more advanced clinical stage, larger tumor size, and lower survival [11]. B7-H3 increases 
radiation resistance of gastric cancer cells by regulating autophagy and affecting apoptosis, cell cycle progression and DNA double-strand break repair [12]. Also, B7-H3 activates the PI3K/AKT signal and up-regulates the protein level of Bcl-2, leading to the sustained growth of ovarian cancer [13]. In renal clear cell carcinoma, the expressions of B7-H3 and tyrosine kinase receptor Tie-2 in tumor vessels are associated with disease progression and prognosis [14]. In addition, inhibition of B7-H3 expression leads to inactivation of P38/MAPK signaling, thereby reducing cell proliferation and glycolysis of metastatic melanoma [15, 16]. But due to the lack of corresponding molecule, the mechanism of action still remained unclear.

Fibronectin (FN) is a multifunctional glycoprotein present in the extracellular matrix (ECM) of plasma and tissues [17]. FN exists in three forms, namely, cellular FN, plasma FN and fetal FN [18]. FN has two disulfide bond-connected subunits with a molecular weight of 220-225 kD. Each subunit contains several ligand-binding domains that activate a series of FN-mediated signal transduction pathways to regulate biological functions, such as cell adhesion, migration, proliferation and embryonic development $[17,19]$.

Adhesion of metastatic cells plays a key role in tumor cell metastasis to host organ [20]. Study showed that the adhesion ability of liver cancer HepG 2 cells decreases after knocking down the expression of B7-H3 [20]. We also found that adherent cells often show high-expressed B7-H3, while suspension cells hardly express $\mathrm{B} 7-\mathrm{H} 3$, which indirectly indicates that B7-H3 may promote cell adhesion.

Previous studies demonstrated that a high expression of $\mathrm{B} 7-\mathrm{H} 3$ or $\mathrm{FN}$ is related to a reduced tumor cell apoptosis, but the interaction between B7-H3 and FN in relation to cell apoptosis has not been investigated. In this study, we aimed to explore whether B7-H3 can regulate the apoptosis of gastric cancer cells through interaction with FN.

\section{Materials and methods}

\section{Cell culture}

MGC-803 and MKN-45 cell lines were purchased from the Cell Bank of the Chinese Academy of Sciences. DMEM high-glucose medium (\#SH30243. 01B, Hyclone, USA) containing $10 \%$ fetal bovine serum (FBS, \#04-001-1ACS, Biological Industries, USA) and 1\% Penicillin and Streptomyces (\#C0222, Beyotime, China) was cultured in a saturated humidity incubator at $37^{\circ} \mathrm{C}$ with $5 \% \mathrm{CO}_{2}$.

\section{Cell transfection and infection}

Silencing of B7-H3 sequences was designed by
Shanghai GeneChem Co., Ltd., with hU6-MCSUbiquitin-EGFP-IRES-puromycin as a plasmid vector. The expression of B7-H3 in cells was knocked down with siRNA of the following target sequences: the sense sequence was 5'-GUGCUGGAGAAAGAUCAA ATT-3', and the antisense sequence was 5'-UUUGA UCUUUCUCCACAGCACTT-3'. According to the instructions, MKN-45 cells and MGC-803 cells were divided into negative control (sh NC) and experimental group (sh B7-H3). 72 hours after the cell transfection, puromycin (\#P8230, Solarbio, China) was added to the cells for two weeks, and then the transfection efficiency was detected by Western blot and $\mathrm{qPCR}$.

\section{Co-immunoprecipitation (Co-IP) assays}

MGC-803 cells and MKN-45 cells were lysed with IP lysis buffer (\#P0013, Beyotime, China). Cell supernatant was collected by centrifugation and reacted with Ig G (\#A7007, Beyotime, China) and Protein A+G Agarose (\#P2055, Beyotime, China) following the instructions. Anti-B7-H3 antibody (\#66481-1-Ig, Proteintech, China) and anti-FN antibody (\#66042-1-Ig, Proteintech, China) were used for Western blot analysis.

\section{Cell adhesion analysis}

The 96-well plate was coated with $20 \mu \mathrm{g} / \mathrm{ml} \mathrm{FN}$ (\#f81801, Solarbio, China), add with 2\% BSA (\#FMS-WB021, Fcmacs, China), and blocked for 1 hour. MGC-803 ctrl-sh NC, MGC-803 FN-sh NC, MGC-803ctrl-sh B7-H3, MGC-803 FN-sh B7-H3 cells were resuspended in 1\% FBS medium and added to a 96-well plate, with each group having 5 replicate wells. After culturing for 1 hour, unadhered cells were removed, while the rest cells were fixed with $4 \%$ paraformaldehyde (\#BL539A, Biosharp, China) and stained with crystal violet (\#C0121, Beyotime, China). Photos were taken for statistical analysis. MKN-45 cell processing was conducted the same as MGC-803 cells.

\section{Apoptosis assays}

The MGC-803 cells were inoculated into a 6-well plate and divided into the following groups: starvation + ctrl-sh NC (cells incubated with serum-free DMEM medium); starvation + FN ctrl-sh NC (cells incubated with serum-free DMEM medium and exogenous FN); starvation + ctrl-sh B7-H3 (cells incubated with serum-free DMEM); starvation + FN ctrl-sh B7-H3 (cells incubated with serum-free DMEM medium and exogenous FN). All the cells were cultured for 24 hours. The Annexin V-PE Apoptosis detect kit (\#559763, BD, USA) was used to detect cell apoptosis. The processing of MKN-45 cells was conducted the same as that of MGC-803 cells. 


\section{Immunohistochemistry staining}

The tissues of 4 mice in the sh NC group and 4 mice in the sh $\mathrm{B} 7-\mathrm{H} 3$ group kept in the tissue fixator (\#KSN00002, China) were dehydrated, embedded with paraffin, and then made into sections. After the antigen was extracted with $10 \mathrm{mM}$ sodium citrate buffer ( $\mathrm{pH}$ 6.0), the sections were incubated with anti-FN antibody (\#66042-1-Ig, Proteintech, China). Following the incubation with the secondary antibody, the tissues were stained with $\mathrm{DAB}$ and counterstained with hematoxylin, dehydrated, mounted on the slides, and then the pictures were taken.

\section{Western blot analysis}

The cultured MGC-803 and MKN-45 cells were collected, washed with PBS, and lysed on ice for 30 min with a RIPA lysis buffer (\#P0013B, Beyotime, China) containing phosphatase inhibitors (\#P1045, Beyotime, China) and protease inhibitors (\#P1005, Beyotime, China). The protein concentrations of the cell lysates were determined by the BCA Protein Assay Kit (\#P0012, Beyotime, China). Protein samples were separated on SDS-PAGE gel and transferred to polyvinylidene diflfluoride (PVDF) membranes (GE Healthcare, Germany), which were then labeled with the following primary antibodies overnight at $4{ }^{\circ} \mathrm{C}$ : anti-B7-H3 (\#66481-1-Ig, Proteintech, China), anti-Fibronectin (\#66042-1-Ig, Proteintech, China), anti-PI3K (\#60225-1-Ig, Proteintech, China), anti-p-AKT (\#66444-1-Ig, Proteintech, China), anti-AKT (\#60203-2-Ig, Proteintech, China), anti-Bax (\#5023,CST,USA), anti-Bcl-2 (\#15071,CST,USA), anti-p-PI3K (\#AF5905, Beyotime, China), anti- $\beta$-actin (\#AF5001, Beyotime, China), anti-Caspase 3 (\#AF1213 Beyotime, China), anti-Caspase 8 (\#AF1243, Beyotime, China), anti-Caspase 9 (\#AF1264, Beyotime, China), anti-Cytochrome C (\#AF2047, Beyotime, China), anti-Apaf-1 (\#AF1462, Beyotime, China), anti-Cleaved PARP (\#AF1567, Beyotime, China) and anti-p53 (\#AF1162, Beyotime, China). After TBST washing, the membranes were incubated with HRP-conjugated secondary antibodies (Beyotime, China), and the signals were detected by using an ECL detection kit (\#36222ES60, Yeasen, China).

\section{RNA extraction and real-time quantitative PCR (qPCR)}

The cells were collected for extracting total RNA using RNA-Quick Purification Kit (\#RN001, Yishan, China). PrimeScript ${ }^{\mathrm{TM}}$ RT Master Mix Kit (\#R0037A, Takara, Japan) and AceQ qPCR SYBR Green Master Mix (without ROX) kit (\#q121, Vazyme, China) were employed to perform real-time quantitative PCR to detect the expression of $\mathrm{B} 7-\mathrm{H} 3$.

\section{Tumor growth in vivo}

Five-week-old SPF BALB/C male nude mice were purchased from Shanghai Experimental Animal Center. All animal experiments were approved by the Institutional Animal Care and Use Committee of Soochow University (Suzhou, China). All the mice were randomly divided into groups. The sh $\mathrm{B} 7-\mathrm{H} 3$ and sh NC MGC-803 cells $\left(5 \times 10^{6}\right)$ were suspended in $150 \mu 1$ PBS at a ratio of $1: 1$ and injected into the subcutaneous layer on the right side of the mice. The tumor volume was recorded every 5 days. After 5 weeks, the xenografts were harvested from the mice. Tumor tissues were immersed in tissue fixative for immunohistochemistry or placed at $-80^{\circ} \mathrm{C}$ for Western blot. All the results of animal experiments were obtained blindly.

\section{Statistical analysis}

The data were obtained from three independent experiment repetitions. Student's t-test was used for comparisons between two groups, and the results were expressed as the mean \pm standard error (mean \pm SEM). $\quad P<0.05$ was considered as statistically significant.

\section{Results}

\section{B7-H3 interacted with FN to promote adhesion of gastric cancer cells}

MGC-803 sh B7-H3 cells and MKN-45 sh B7-H3 cells were successfully constructed for subsequent experiments (Fig. S1). In B7-H3 low group (MGC-803), the cell adhesion rate was significantly reduced when compared with that in B7-H3 high group (MGC-803). After exogenous FN treatment, the cell adhesion rate of B7-H3high group (MGC-803) became greatly higher than B7-H3low group (MGC-803), and the cell adhesion rate of $\mathrm{B} 7-\mathrm{H} 3^{\text {low }}$ group (MGC-803) and B7-H3high group (MGC-803) was increased noticeably with in addition of exogenous FN (Fig. 1A). The similar results were obtained in MKN-45 cells (Fig. 1B). Based on the above results, we found that in gastric cancer cells, B7-H3 bound to FN to promote cell adhesion.

\section{B7-H3/FN interaction inhibited gastric cancer cell apoptosis}

As shown in Fig. 2A, early apoptosis of the cells in B7-H3 high group (MGC-803) was significantly reduced, but there was no significant change in the late apoptosis after exogenous FN treatment. In B7-H3low group (MGC-803), there was no significant change in early or late apoptosis with the addition of exogenous FN. The similar results were also found in MKN-45 cells (Fig. 2B). 

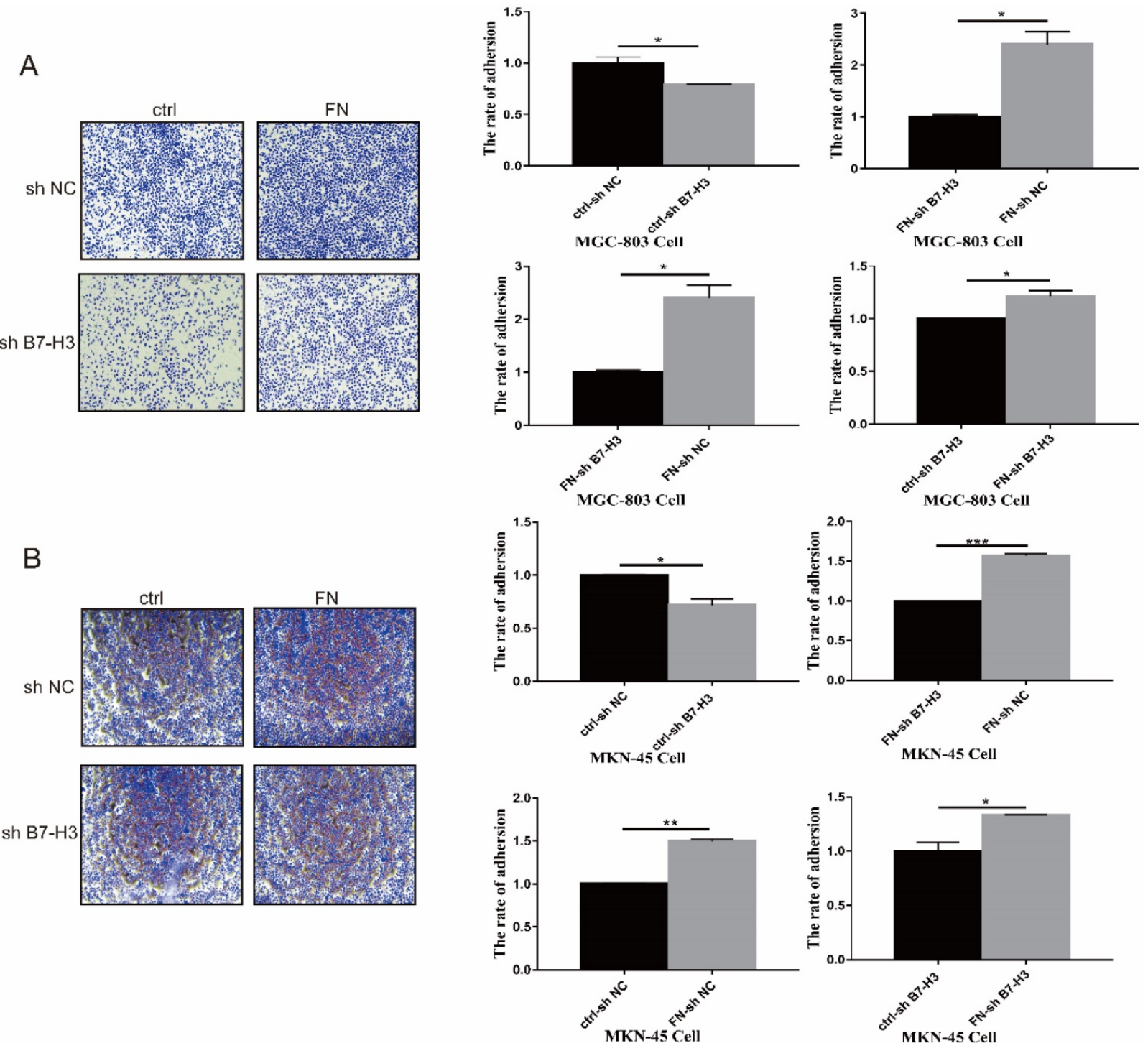

Figure 1. B7-H3 interacted with FN to promote cell adhesion. B7-H3 interacted with FN to promote the cell adhesion of MGC-803 cells (A) and MKN-45 cells (B). * $<0.05$, $* * \mathrm{p}<0.01, * * * \mathrm{p}<0.001$.

Subsequently, we examined the changes in apoptotic proteins. In MGC-803 cells, Cleaved PARP, Caspase 8 and Caspase 9 expressions were significantly decreased in the FN-sh NC group after exogenous FN stimulation compared with the ctrl-sh NC group $(P<0.05)$, Cytochrome $C$ expression increased $(P<0.05)$, but apaf-1 expression had no significant change $(P>0.05)$; Cleaved PARP and Cytochrome $C$ expression were not significantly changed $(P>0.05)$, Caspase 8 and Caspase 9 expression were significantly decreased $(P<0.05)$ in the FN-sh B7-H3 group compared with the ctrl-sh B7-H3 group after exogenous FN stimulation, the expression of apaf-1 was increased $(\mathrm{P}<0.05)$, but the decrease of Caspase 9 expression in ctrl-sh B7-H3 group was less than that in ctrl-sh NC group after adding exogenous FN(Fig. 2C), and similar results were obtained in MKN-45 cells (Fig. 2D). These results suggest that exogenous FN and B7-H3 may inhibit the apoptosis of gastric cancer cells through Caspase 9.

\section{The interaction between $\mathrm{B} 7-\mathrm{H} 3$ and $\mathrm{FN}$ in gastric cancer cells}

Correlation between $\mathrm{B} 7-\mathrm{H} 3$ and $\mathrm{FN}$ in gastric cancer was identified from the TCGA database (Fig. 3A). We found that FN polyclonal antibodies (\#AF1918, R\&D, USA) can recognize FN and B7-H3 (data not shown), suggesting that FN and B7-H3 may interact with each other through the formation of natural complexes.

FN was co-precipitated by B7-H3 in MGC-803 and MKN-45 cells, and this further confirmed their interaction between B7-H3 and FN (Fig. 3B). Based on the above results, it can be found that $\mathrm{B} 7-\mathrm{H} 3$ and $\mathrm{FN}$ bind to each other in MGC-803 and MKN-45 cells. 

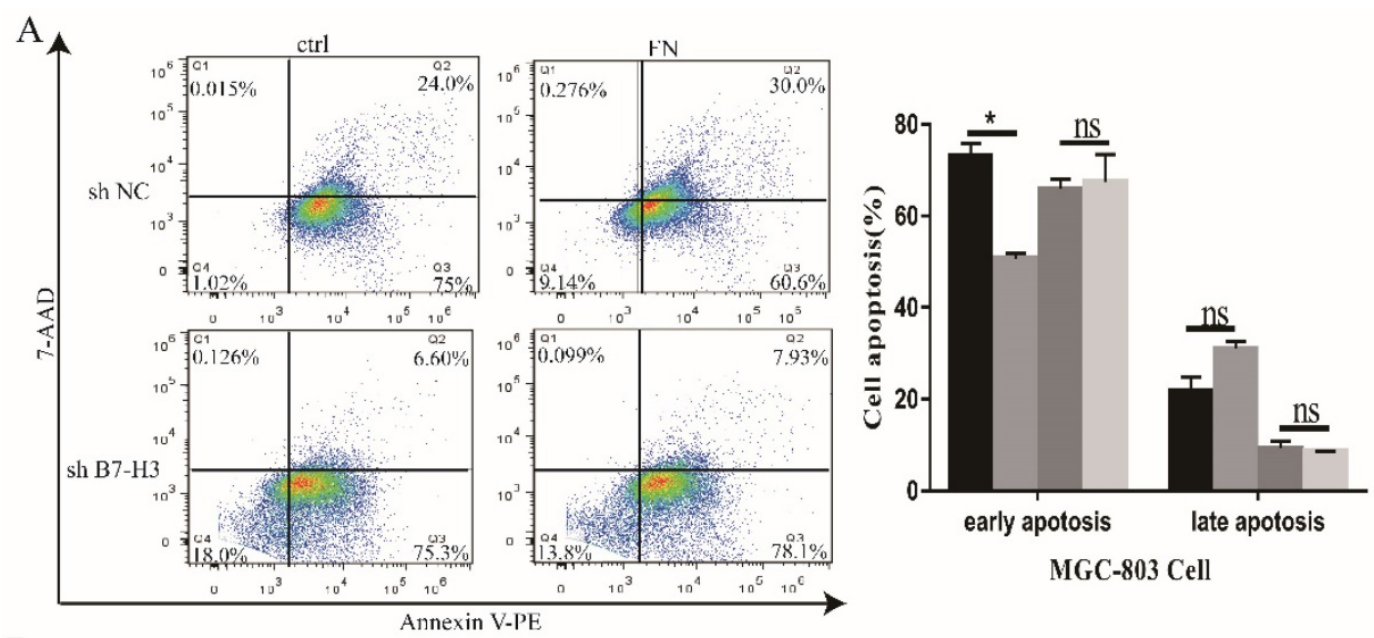

ctrl-sh NC

FN-sh NC

ctrl-sh B7-H3

FN-sh B7-H3
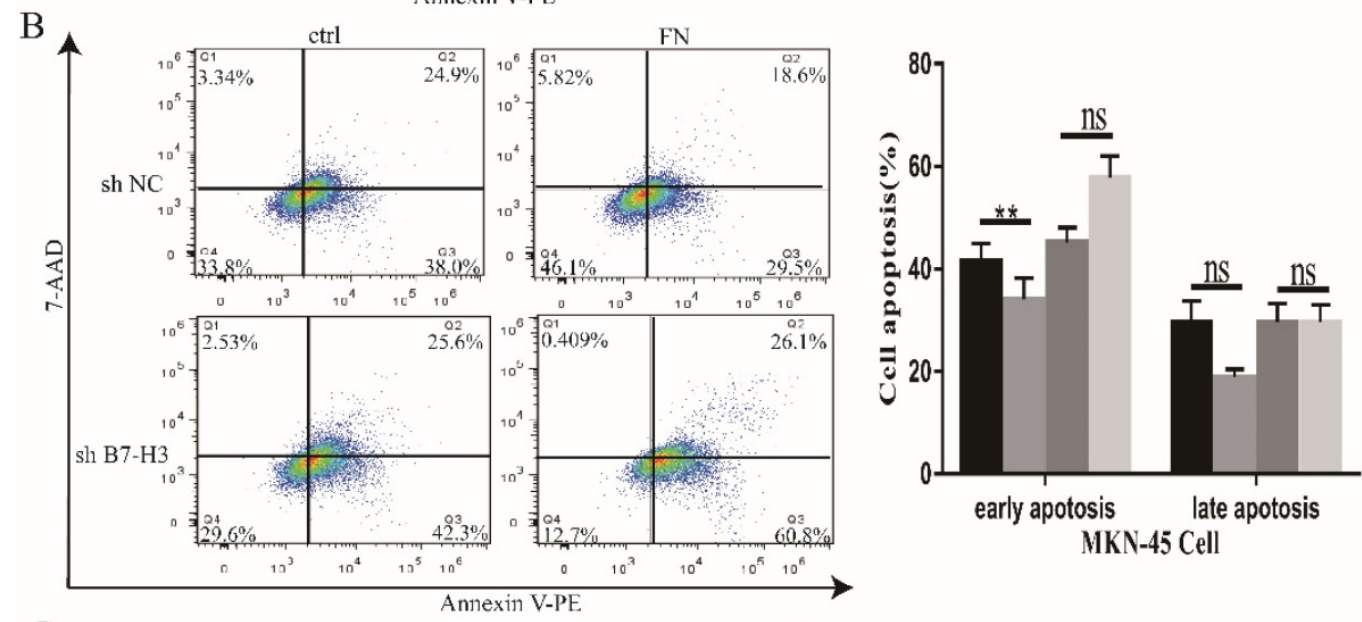

ctrl-sh NC

FN-sh NC

ctrl-sh B7-H3

FN-sh B7-H3

C
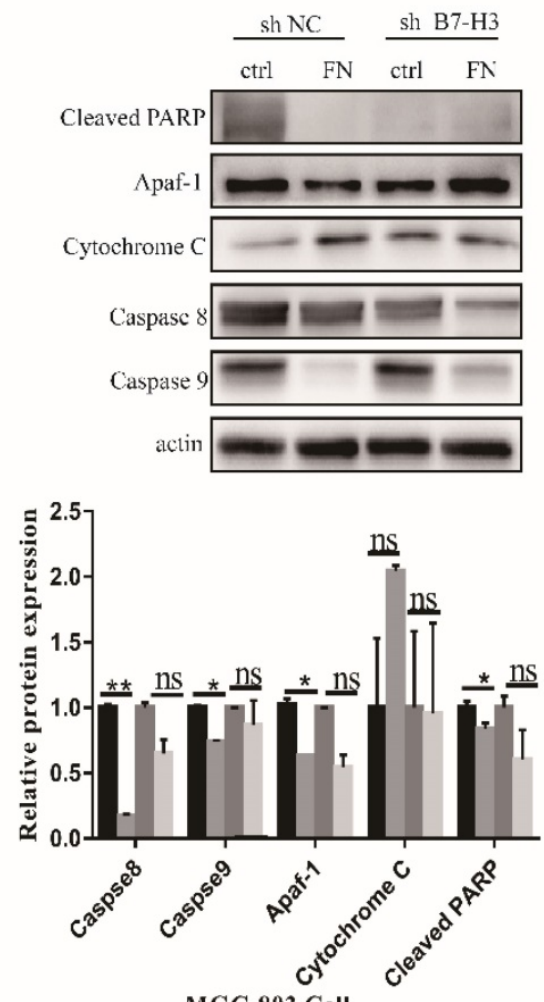

D

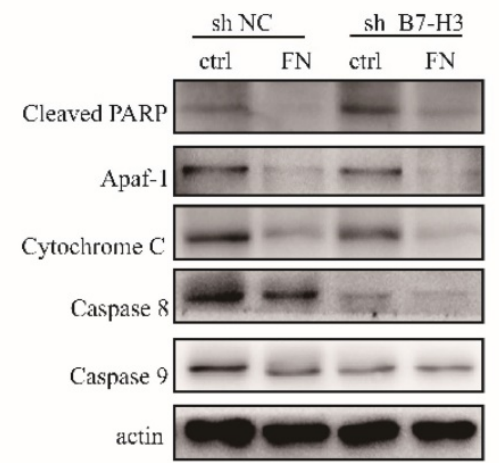

ctrl-sh NC

- FN-sh NC

ctrl-sh B7-H3

FN-sh B7-H3

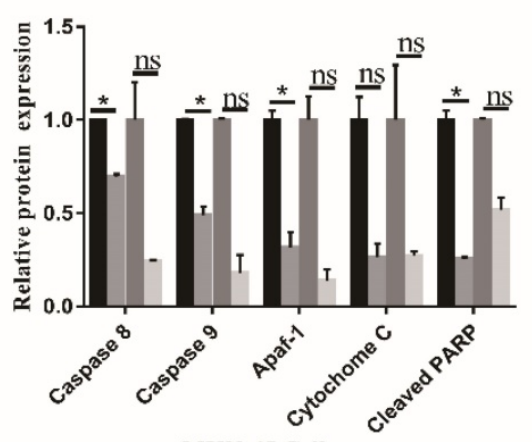

ctrl-sh NC FN--sh NC ctrl-sh B7-II3 FN-sh B7-H3

Figure 2. B7-H3/FN interaction inhibited the apoptosis of gastric cancer cells and down-regulated the expression of apoptotic protein. B7-H3/FN interaction inhibited the apoptosis of gastric cancer MGC-803 cells (A) and MNK-45 cells (B). Changes in the expressions of apoptotic proteins in MGC-803 cells (C) and MKN-45 cells (D). * ${ }^{2}<0.05$, ** $\mathrm{p}<0.01$, *** $\mathrm{p}<0.001$. 
A

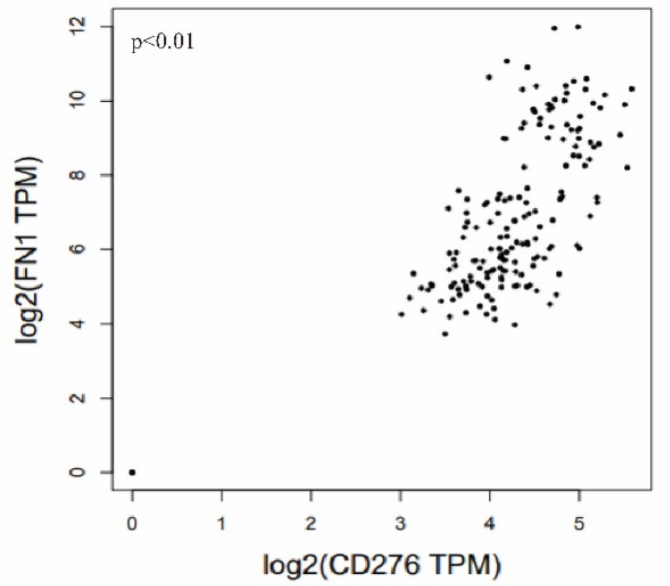

B

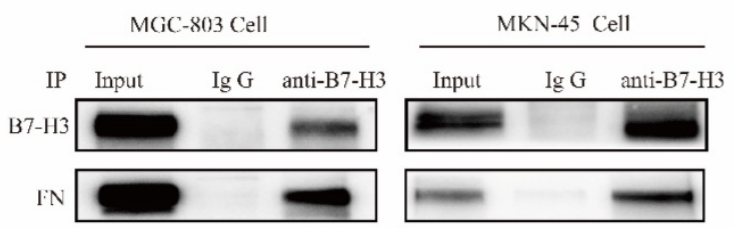

Figure 3. Detection of the interaction between B7-H3 and FN. (A)The correlation coefficients of B7-H3 and FN genes were analyzed by TCGA database; (B) Co-IP assay were used to detect the interaction between $\mathrm{B} 7-\mathrm{H} 3$ and $\mathrm{FN} .{ }^{*} \mathrm{p}<0.05,{ }^{* *} \mathrm{p}<0.01,{ }^{*} *{ }^{*} \mathrm{p}<0.001$.

A
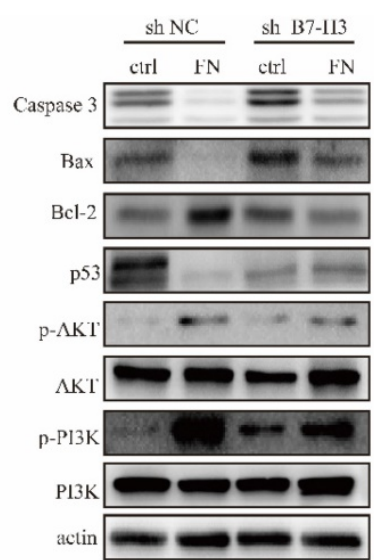

B

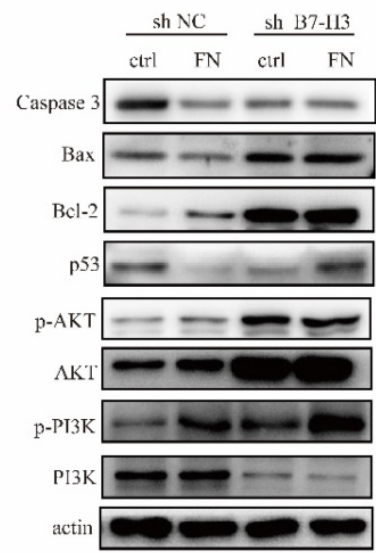

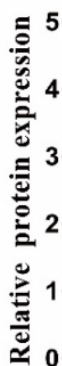

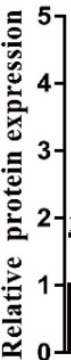

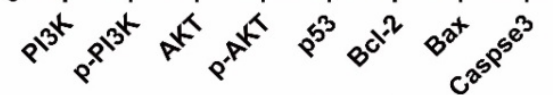

MGC-803 Cell rl-sh NC

$\mathrm{FN}$-sh NC

ctrl-sh B7-H3

FN-sh B7-H3

ctrl-sh NC

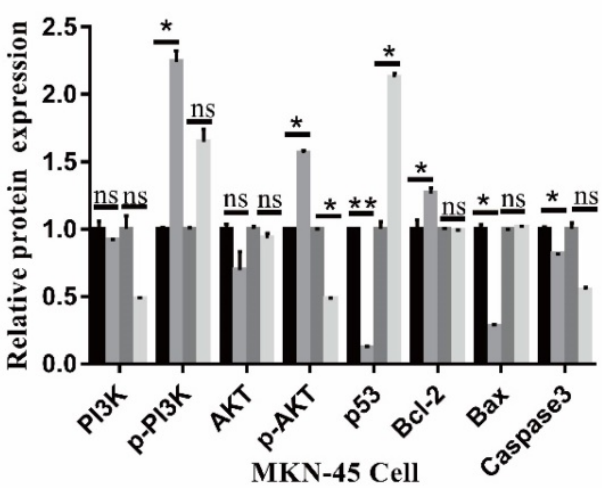

FN-sh NC

ctrl-sh B7-H3

FN-sh B7-H3

Figure 4. B7-H3/FN interaction may inhibit the apoptosis of gastric cancer cells by activating the PI3K/AKT signaling pathway. B7-H3/FN interaction may activate the expression of PI3K/AKT signaling pathway in MGC-803 cells $(\mathbf{A})$ and MKN-45 cells $(\mathbf{B}) .{ }^{*} p<0.05,{ }^{*} \mathrm{p}<0.01, * * * p<0.001$.

\section{B7-H3/FN interaction can activate PI3K/AKT signaling pathway and inhibit the apoptosis of gastric cancer cells}

In MGC-803 cells, the expressions of signal molecular p-PI3K, p-AKT and anti-apoptotic protein Bcl-2 were significantly up-regulated, whereas the expression levels of pro-apoptotic protein p53, Bax and Caspase 3 were sharply down-regulated after adding exogenous FN in ctrl-sh NC group. In ctrl-sh B7-H3 group, the expression levels of these protein molecules did not change with the addition of exogenous FN (Fig. 4A), and the similar results were obtained in MKN-45 cells (Fig. 4B). These experimental results indicated that $\mathrm{B} 7-\mathrm{H} 3 / \mathrm{FN}$ interaction can inhibit the apoptosis of gastric cancer cells by activating the PI3K/AKT signaling pathway. 

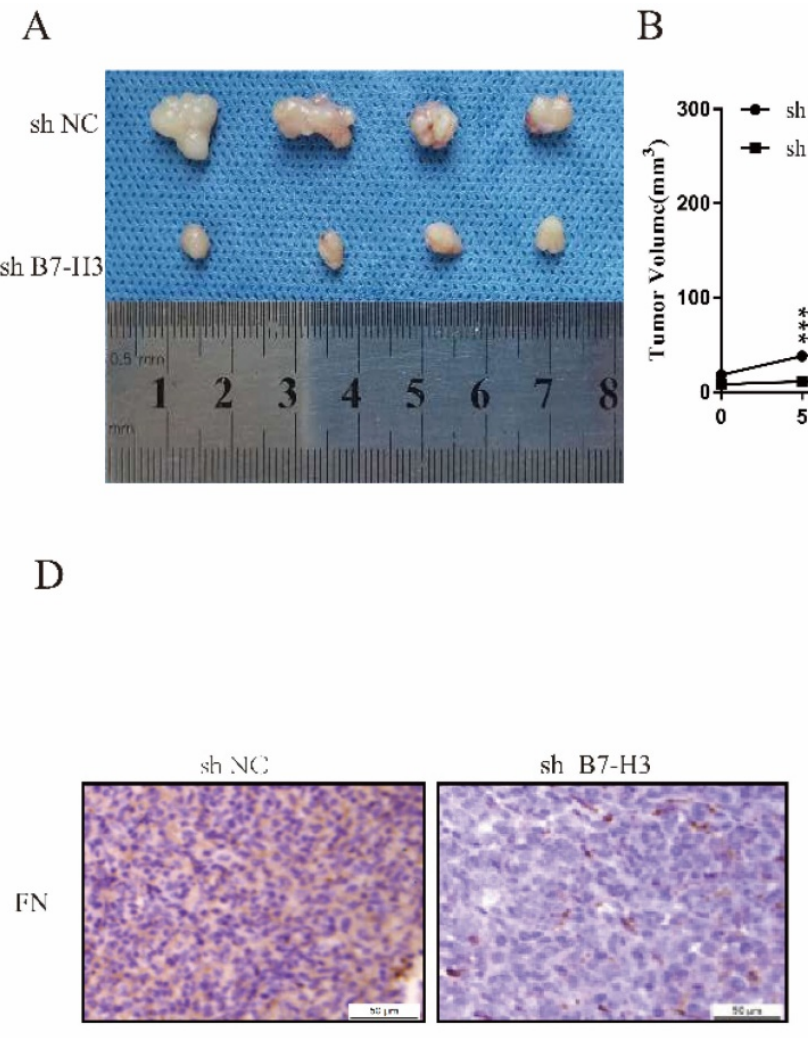

D
B

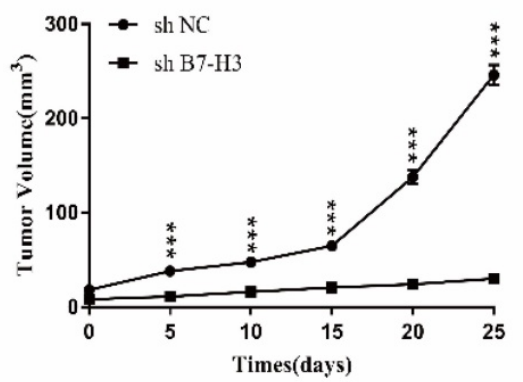

C

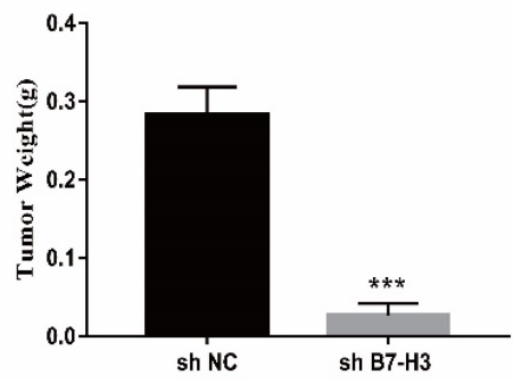

E
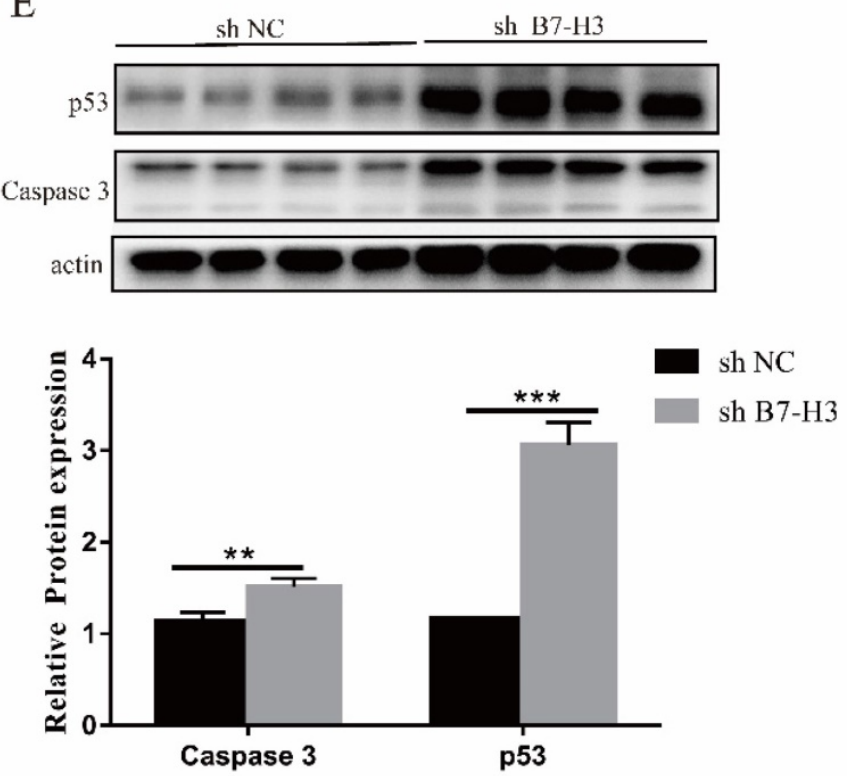

Figure 5. B7-H3 could inhibit growth of tumor in vivo. The size, volume and weight of the tumor after silencing the expression of B7-H3 (A-C). Immunohistochemistry to detect the levels of FN in mouse tumors. Scale bar, $50 \mu \mathrm{m}(\mathbf{D})$. Western blot was used to detect the expressions of $\mathrm{p} 53$ and Caspase 3 in mouse tumors(E). ${ }^{*} p<0.05$, $* * p<0.01$, $* * * p<0.001$.

\section{Silencing the expression of B7-H3 could inhibit tumor growth in vivo}

Due to the short action time of FN, only sh NC and sh B7-H3 cells of MGC-803 cells were used to establish mouse xenograft tumor models. We found that in sh B7-H3 group, the tumor size, volume and weight were all reduced to varying degrees (Fig. 5A-C). Subsequently, it was also found that compared with the sh NC group, the FN level in the sh B7-H3 group was decreased (Fig. 5D), and this showed that the adsorbed FN level was decreased, suggesting that the binding of B7-H3 and FN may be caused by sedimentation. At the same time, we also detected the expressions of apoptotic proteins p53 and Caspase 3, and observed that in sh $\mathrm{B} 7-\mathrm{H} 3$ group, the expressions of p53 and Caspase 3 were significantly up-regulated (Figure 5E). Our results showed that silencing the expression of $\mathrm{B} 7-\mathrm{H} 3$ promoted the expression of apoptotic proteins.

\section{Discussion}

As an immunomodulatory factor, the role of B7-H3 varies in different types of human tumors, such as gastric cancer, kidney cancer, and colorectal cancer [21]. B7-H3 regulates cancer-related signaling pathways in the non-immune system to affect tumor progression [6, 20]. Chen et al. found that hB7-H3 molecule has an effect on the adhesion of extracellular matrix, such as FN, collagenIV and laminin, and its mediated adhesion can effectively promote the adhesion of tumor cells, but the direct binding of B7-H3 molecule to FN and its functional mechanism was not confirmed [22]. In this study, we preliminarily explored the regulatory relationship of the interaction between $\mathrm{B} 7-\mathrm{H} 3$ and $\mathrm{FN}$ on cell adhesion. Our experimental results showed that B7-H3 interacted with FN, and that the interaction of B7-H3 and FN could effectively promote MGC-803 and MKN-45 cell adhesion. Previous studies 
demonstrated that cell adhesion can affect tumor cell proliferation, migration, apoptosis and other processes, and that cell adhesion to extracellular matrix such as FN may reduce its resistance to chemical drugs [23-26].

Apoptosis is an active and orderly cell death regulated by genes. In the presence of ATP or dATP, Cytochrome $\mathrm{C}$ will combine with Apaf-1 to form a complex that activates Caspase-9, thereby activating Caspase-3 and cleaving the substrate to induce apoptosis [27]. Bcl-2 prevents apoptosis by preventing pro-apoptotic molecules from entering the cytoplasm from mitochondria, while Bax promotes apoptosis by inducing mitochondrial outer membrane permeability [28-30]. The ratio of Bax/Bcl-2 determines the sensitivity of cells to death signal [31]. Apaf-1 plays an important role in the mitochondrial apoptotic pathway, because it enhances a variety of processes including the formation of apoptotic bodies with Cytochrome C and dATP, and can activate Caspase- 3 and induce cell apoptosis [32]. Caspase 8 is a key mediator of adhesion cell death [33]. In our present study, we found that in sh NC group, exogenous FN significantly inhibited cell apoptosis, while in sh B7-H3 group, apoptosis did not show great change after the addition of exogenous FN. In addition, we found that in sh NC group, exogenous FN sharply down-regulated the expressions of pro-apoptotic proteins Caspase 8, Caspase 9, Apaf-1 and Cleaved PARP, while in sh B7-H3 group, the expressions of pro-apoptotic proteins Caspase 8, Caspase 9, apaf-1 and Cleaved PARP did not change greatly after addition of exogenous FN. These data suggested that B7-H3 inhibited apoptosis of gastric cancer cells through interacting with FN.

More evidence showed that extracellular matrix adhesion activates the PI3K/AKT signaling pathway, thereby inhibiting various forms of tumor cell death $[34,35]$. PI3K is a key regulator of the formation of adhesion junction, and it can directly bind to E-Cadherin, thereby regulating adhesion-mediated cell cycle progression [36, 37]. Studies have also shown that down-regulation of AKT protein expression can significantly induce cell apoptosis and inhibit cell proliferation [38]. It has been determined that the downstream target proteins of the AKT signaling pathway are protein molecules related to apoptosis, such as Bax, Bcl-2 and Caspase 3 [39]. Activated AKT may affect cell cycle progression and tumor growth via the p53 pathway and $\mathrm{Bcl}-2$ protein family [40]. In this research, we found that in sh NC group, the expressions of p-PI3K, p-AKT and Bcl-2 were up-regulated, and the expressions of p53, Bax and Caspase 3 were down-regulated following the treatment of exogenous FN. However, in sh B7-H3 group, the expression of these proteins did not change significantly after the addition of exogenous FN. Thus, B7-H3 inhibited the apoptosis of gastric cancer cells and activated PI3K/AKT signaling pathway through interacting with FN.

In vivo studies indicated that $\mathrm{B} 7-\mathrm{H} 3$ plays an important role in tumor progression. Previous report also proved that FN has a certain correlation with cell proliferation [41]. Our experimental results showed that low-expressed B7-H3 inhibited the growth of mouse tumors, and that the level of FN was also reduced. At the same time, the expressions of p53 and Caspase 3 were up-regulated.

In conclusion, we found that B7-H3 inhibited the apoptosis of gastric cancer cell through interacting with $\mathrm{FN}$, and that the related signaling pathway may be PI3K/AKT. These experimental results indicated that adhesion and PI3K/AKT signaling pathway is possibly correlated, and may be a potential target for tumor therapy in the future.

\section{Supplementary Material}

Supplementary figure.

https://www.jcancer.org/v12p7518s1.pdf

\section{Acknowledgements}

\section{Ethical approval statement}

All experimental procedures used in this study were performed in accordance with the approved guidelines of the ethical board of Soochow University College of Medicine.

\section{Funding}

This study was supported by the National Natural Science Foundation of China (NO.81872328) and Major Project of Natural Science Research of Jiangsu Provincial Department of Education (NO.17KJA310004).

\section{Competing Interests}

The authors have declared that no competing interest exists.

\section{References}

1. Molina-Castro S, Pereira-Marques J, Figueiredo C, Machado JC, Varon C: Gastric cancer: Basic aspects. Helicobacter 2017, 22 Suppl 1:12412-12419.

2. Wang Z, Wang Z, Zhang C, Liu X, Li G, Liu S, Sun L, Liang J, Hu H, Liu Y et al: Genetic and clinical characterization of B7-H3 (CD276) expression and epigenetic regulation in diffuse brain glioma. Cancer science 2018, 109(9):2697-2705.

3. Chapoval AI, Ni J, Lau JS, Wilcox RA, Flies DB, Liu D, Dong H, Sica GL, Zhu G, Tamada K et al: B7-H3: a costimulatory molecule for T cell activation and IFN-gamma production. Nature immunology 2001, 2(3):269-274.

4. Ni L, Dong C: New B7 Family Checkpoints in Human Cancers. Molecular cancer therapeutics 2017, 16(7):1203-1211.

5. Liu C, Liu J, Wang J, Liu Y, Zhang F, Lin W, Gao A, Sun M, Wang Y, Sun Y: B7-H3 expression in ductal and lobular breast cancer and its association with IL-10. Molecular medicine reports 2013, 7(1):134-138.

6. Wang L, Kang FB, Shan BE: B7-H3-mediated tumor immunology: Friend or foe? International journal of cancer 2014, 134(12):2764-2771. 
7. Castriconi R, Dondero A, Augugliaro R, Cantoni C, Carnemolla B, Sementa AR, Negri F, Conte R, Corrias MV, Moretta L et al: Identification of 4Ig-B7-H3 as a neuroblastoma-associated molecule that exerts a protective role from an NK cell-mediated lysis. Proceedings of the National Academy of Sciences of the United States of America 2004, 101(34):12640-12645.

8. Greenwald RJ, Freeman GJ, Sharpe AH: The B7 family revisited. Annual review of immunology 2005, 23:515-548.

9. Wu S, Zhao X, Wu S, Du R, Zhu Q, Fang H, Zhang X, Zhang C, Zheng W, Yang $\mathrm{J}$ et al: Overexpression of B7-H3 correlates with aggressive clinicopathological characteristics in non-small cell lung cancer. Oncotarget 2016, 7(49):81750-81756.

10. Picarda E, Ohaegbulam KC, Zang X: Molecular Pathways: Targeting B7-H3 (CD276) for Human Cancer Immunotherapy. Clinical cancer research : an official journal of the American Association for Cancer Research 2016, 22(14):3425-3431.

11. Chen JT, Chen $\mathrm{CH}, \mathrm{Ku} \mathrm{KL}, \mathrm{Hsiao} \mathrm{M}$, Chiang $\mathrm{CP}, \mathrm{Hsu} \mathrm{TL}$, Chen MH, Wong $\mathrm{CH}$ : Glycoprotein $\mathrm{B} 7-\mathrm{H} 3$ overexpression and aberrant glycosylation in oral cancer and immune response. Proceedings of the National Academy of Sciences of the United States of America 2015, 112(42):13057-13062.

12. Li Y, Yang X, Yao P, Shen W, Wu Y, Ye Z, Zhao K, Chen H, Cao J, Xing C: $\mathrm{B} 7-\mathrm{H} 3$ increases the radioresistance of gastric cancer cells through regulating baseline levels of cell autophagy. American journal of translational research 2019, 11(7):4438-4449.

13. Zhou L, Zhao Y: B7-H3 Induces Ovarian Cancer Drugs Resistance Through An PI3K/AKT/BCL-2 Signaling Pathway. Cancer management and research 2019, 11:10205-10214

14. Zhang X, Ji J, Zhang G, Fang C, Jiang F, Ma S, Hou J: Expression and significance of $\mathrm{B} 7-\mathrm{H} 3$ and Tie-2 in the tumor vasculature of clear cell renal carcinoma. OncoTargets and therapy 2017, 10:5417-5424.

15. Flem-Karlsen K, Tekle C, Andersson Y, Flatmark K, Fodstad Ø, Nunes-Xavier CE: Immunoregulatory protein $\mathrm{B} 7-\mathrm{H} 3$ promotes growth and decreases sensitivity to therapy in metastatic melanoma cells. Pigment cell \& melanoma research 2017, 30(5):467-476.

16. Flem-Karlsen K, Tekle C, Øyjord T, Flørenes VA, Mælandsmo GM, Fodstad $\varnothing$, Nunes-Xavier CE: p38 MAPK activation through B7-H3-mediated DUSP10 repression promotes chemoresistance. Scientific reports 2019, 9(1):5839-5848.

17. White ES, Baralle FE, Muro AF: New insights into form and function of fibronectin splice variants. The Journal of pathology 2008, 216(1):1-14.

18. Singh P, Carraher C, Schwarzbauer JE: Assembly of fibronectin extracellular matrix. Annual review of cell and developmental biology 2010, 26:397-419.

19. To WS, Midwood KS: Plasma and cellular fibronectin: distinct and independent functions during tissue repair. Fibrogenesis \& tissue repair 2011, 4:21-38.

20. Wang F, Wang G, Liu T, Yu G, Zhang G, Luan X: B7-H3 was highly expressed in human primary hepatocellular carcinoma and promoted tumor progression. Cancer investigation 2014, 32(6):262-271.

21. Li G, Quan Y, Che F, Wang L: B7-H3 in tumors: friend or foe for tumor immunity? Cancer chemotherapy and pharmacology 2018, 81(2):245-253.

22. Chen YW, Tekle C, Fodstad O: The immunoregulatory protein human B7H3 is a tumor-associated antigen that regulates tumor cell migration and invasion. Current cancer drug targets 2008, 8(5):404-413.

23. Lechuga S, Amin PH, Wolen AR, Ivanov AI: Adducins inhibit lung cancer cell migration through mechanisms involving regulation of cell-matrix adhesion and cadherin-11 expression. Biochimica et biophysica acta Molecular cell research 2019, 1866(3):395-408.

24. Westhoff MA, Fulda S: Adhesion-mediated apoptosis resistance in cancer. Drug resistance updates : reviews and commentaries in antimicrobial and anticancer chemotherapy 2009, 12(4-5):127-136.

25. Pan CW, Shen ZJ, Wu TT, Tang XY, Wang M, Sun J, Shao Y: Cell adhesion to fibronectin induces mitomycin $\mathrm{C}$ resistance in bladder cancer cells. BJU international 2009, 104(11):1774-1779.

26. Januskevicius A, Janulaityte $\mathrm{I}$ Kalinauskaite-Zukauske $\mathrm{V}$, Gosens $\mathrm{R}$ Malakauskas K: The Enhanced Adhesion of Eosinophils Is Associated with Their Prolonged Viability and Pro-Proliferative Effect in Asthma. Journal of clinical medicine 2019, 8(9):1274-1297.

27. Zhu B, Wei Y: Antitumor activity of celastrol by inhibition of proliferation, invasion, and migration in cholangiocarcinoma via PTEN/PI3K/Akt pathway. Cancer medicine 2020, 9(2):783-796.

28. Karlsson H, Lindqvist AC, Fransson M, Paul-Wetterberg G, Nilsson B, Essand M, Nilsson K, Frisk P, Jernberg-Wiklund H, Loskog A: Combining CAR T cells and the Bcl-2 family apoptosis inhibitor ABT-737 for treating B-cell malignancy. Cancer gene therapy 2013, 20(7):386-393.

29. Wang $X$ : The expanding role of mitochondria in apoptosis. Genes \& development 2001, 15(22):2922-2933.

30. Tait SW, Green DR: Mitochondria and cell death: outer membrane permeabilization and beyond. Nature reviews Molecular cell biology 2010, 11(9):621-632.

31. Zhang S, Zhou C, Zhang D, Huang Z, Zhang G: The anti-apoptotic effect on cancer-associated fibroblasts of B7-H3 molecule enhancing the cell invasion and metastasis in renal cancer. OncoTargets and therapy 2019, 12:4119-4127.

32. Yun T, Yu K, Yang S, Cui Y, Wang Z, Ren H, Chen S, Li L, Liu X, Fang M et al: Acetylation of p53 Protein at Lysine 120 Up-regulates Apaf-1 Protein and Sensitizes the Mitochondrial Apoptotic Pathway. The Journal of biological chemistry 2016, 291(14):7386-7395
33. Stupack DG, Puente XS, Boutsaboualoy S, Storgard CM, Cheresh DA: Apoptosis of adherent cells by recruitment of caspase- 8 to unligated integrins. The Journal of cell biology 2001, 155(3):459-470.

34. Hodkinson PS, Elliott T, Wong WS, Rintoul RC, Mackinnon AC, Haslett C, Sethi T: ECM overrides DNA damage-induced cell cycle arrest and apoptosis in small-cell lung cancer cells through beta1 integrin-dependent activation of PI3-kinase. Cell death and differentiation 2006, 13(10):1776-1788.

35. Aoudjit F, Vuori K: Integrin signaling inhibits paclitaxel-induced apoptosis in breast cancer cells. Oncogene 2001, 20(36):4995-5004.

36. De Santis G, Miotti S, Mazzi M, Canevari S, Tomassetti A: E-cadherin directly contributes to $\mathrm{PI} 3 \mathrm{~K} / \mathrm{AKT}$ activation by engaging the PI3K-p85 regulatory subunit to adherens junctions of ovarian carcinoma cells. Oncogene 2009, 28(9):1206-1217.

37. Rivard N: Phosphatidylinositol 3-kinase: a key regulator in adherens junction formation and function. Frontiers in bioscience (Landmark edition) 2009, 14:510-522.

38. Yuan Y, Du W, Wang Y, Xu C, Wang J, Zhang Y, Wang H, Ju J, Zhao L, Wang $\mathrm{Z}$ et al: Suppression of AKT expression by miR-153 produced anti-tumor activity in lung cancer. International journal of cancer 2015, 136(6):1333-1340.

39. Shi M, Zhang H, Li M, Xue J, Fu Y, Yan L, Zhao X: Normal endometrial stromal cells regulate survival and apoptosis signaling through $\mathrm{PI} 3 \mathrm{~K} / \mathrm{AKt} /$ Survivin pathway in endometrial adenocarcinoma cells in vitro. Gynecologic oncology 2011, 123(2):387-392.

40. Lin W, Xie J, Xu N, Huang L, Xu A, Li H, Li C, Gao Y, Watanabe M, Liu C et al: Glaucocalyxin A induces G2/M cell cycle arrest and apoptosis through the PI3K/Akt pathway in human bladder cancer cells. International journal of biological sciences 2018, 14(4):418-426.

41. Yi W, Xiao E, Ding R, Luo P, Yang Y: High expression of fibronectin is associated with poor prognosis, cell proliferation and malignancy via the $\mathrm{NF}-\mathrm{kB} / \mathrm{p} 53$-apoptosis signaling pathway in colorectal cancer. Oncology reports 2016, 36(6):3145-3153. 